\title{
Consensus Problems in Multiagent Systems with Event-Triggered Dynamic Quantizers
}

\author{
Changgeng Luo, ${ }^{1}$ Xinsheng Wang, ${ }^{2}$ and Yu Ren ${ }^{3}$ \\ ${ }^{1}$ Department of Physics \& Electronic Engineering College, Nanyang Normal University, Nanyang 473000, China \\ ${ }^{2}$ School of Information and Electrical Engineering, Harbin Institute of Technology at Weihai, Weihai 264209, China \\ ${ }^{3}$ School of Astronautics, Harbin Institute of Technology, Harbin 150001, China
}

Correspondence should be addressed to Xinsheng Wang; wangxswh@126.com

Received 19 December 2013; Accepted 3 March 2014; Published 3 April 2014

Academic Editor: Xin Wang

Copyright (C) 2014 Changgeng Luo et al. This is an open access article distributed under the Creative Commons Attribution License, which permits unrestricted use, distribution, and reproduction in any medium, provided the original work is properly cited.

Considering the constrained communication data rate and compute capability that commonly exists in multiagent systems, this paper modifies a current consensus strategy by introducing a kind of dynamic quantizer in both state feedback and control input and updates dynamic quantizers by employing event-triggered strategies, thus forming a new quantitative consensus strategy. The numerical simulation example is built for state quantization and the results show the consistency with expectation.

\section{Introduction}

Control theories for a single agent have been studied over a century; many strategies have been developed maturely, for example, robust control [1,2], fractional-order control $[3,4]$, fuzzy control and neural networks $[5,6]$, and so forth. At the same time, the systems are becoming more and more complex which lead to the increasing cost in analyzing and controlling available systems. Researches on multiagent systems have raised much attention. Liu and Geng study the finite-time optimal formation control problem for secondorder multiagent systems in [7]; meanwhile the formation time and/or the cost function must be considered in the study. Consensus problem is a basic and most important direction in the study of multiagent systems [8-10]. Communications among agents are always wireless, which means that the energy of each agent is also limited and the communication data rate among agents are always constrained. This motivates the employment of quantizers in multiagent systems.

Fortunately, Ceragioli, Persis and Frasca in problem of quantization used in single agent has been studied for a long time, and many kinds of quantizers have been designed and well used to solve problems. In [11], the problem of quantized filtering in linear stochastic systems is investigated. In [12], logarithmic quantizers are used to attain the minimum data rate in stabilizing linear systems. A number of quantized feedback controllers for linear systems are designed by $\mathrm{Fu}$ and Xie in [13]. Quantizers are also well used to solve some other problems, as [14-16] presented. But quantizers in these researches are all with fixed parameters, which constrain their application seriously. In [17], Liberzon introduces a kind of dynamic quantizer with a variable parameter to stabilize a hybrid feedback system.

What is more, the convergence time of multiagent systems with quantizers is always finite. Motivated by this and the successful application of quantizers in single agent, quantization consensus problems of multiagent systems attract more and more attention recently. In [18], only the signs of the relative state measurement, which could be regarded as quantization to some extent, are used to achieve finitetime distributed consensus. Further studies about quantized consensus can be found in [19]. A hysteretic quantizer is used to cope with the undesired chattering phenomena in average consensus problem of multiagent systems by [20] and so forth. Think about the reality that knowledge about the upper bounds of the agents' states is always unknown previously. Taking the constrained communication data rate and limited compute resources into consideration, this paper 
employs a kind of dynamic quantizer to revise a kind of consensus strategy, which appears in [21]. To relieve the compute burden of the control unit embedded in each agent, this paper proposed a centralized event-triggered method to update the dynamic parameters of the quantizers. The time upper bound is also given to guarantee the occurrence of the proposed centralized event. Then theorems are given to show the steps of the proposed consensus strategies. A numerical example illustrate the consensus performance of the state quantization case, and the simulation results show the consensus strategy proposed in this paper works well for the considered systems.

The rest of this paper is organized as follows: Section 2 gives some preliminary knowledge about graph theory and quantizer. Section 3 presents the problems we studied. Section 4 presents the results we obtain for both state quantization and input quantization cases. Section 5 gives an example of state quantization case. Finally, some conclusions are drawn in Section 6.

\section{Preliminaries}

2.1. Graph Theory. An undirected graph $G=(\ell, \varepsilon, A)$ will be used to model the communication topologies among $N$ agents; $\ell=\{1,2, \ldots, N\}$ denotes the set of $N$ agents; each agent is called a node; $\varepsilon \in \ell \times \ell$ is the set of edges, where $(i, j) \in \varepsilon$ means the length of the path between agent $i$ and agent $j$ is 1 ; note that the path is undirected; $A=\left(a_{i j}\right)$ is a $N \times N$ matrix given by $a_{i j}=1$ if $(i, j) \in \varepsilon$, and $a_{i j}=0$ if $(i, j) \notin \varepsilon$. A sequence of distinct nodes from agent $i$ to agent $j$, where the consecutive nodes are adjacent, is called a path. A path of length $r$ means there are $r+1$ nodes in the path, a path that is a cycle if $i=j . G$ is called connected which means there is a path between any two nodes of it.

The Laplacian matrix $L=\left(l_{i j}\right)$ of graph $G$ is defined by $l_{i j}=-1$ for $i \neq j$ with $(i, j) \in \varepsilon$, and $l_{i i}=\sum_{k=1, k \neq i}^{n} a_{i k}$. A connected graph satisfies the eigenvalues of its Laplacian matrix has one zero (which is smaller than any other eigenvalues), and the corresponding eigenvector is of ones.

2.2. Quantizer. The variable quantized is denoted by $k \in R^{l}$. A quantizer can be regarded as a map $q$ from set $R^{l}$ to set $\hbar$, where set $\hbar$ is a finite set, $\hbar \subset R^{l}$. That is, if $k \in R^{l}$, then $q(k) \in \hbar$. A quantization region is a range of $k ; q(k)$ remains constant if $k$ is in this region. Note that the shape of the regions is arbitrary. A quantization interval $\Delta$ is the distance between two consecutive quantization values. If $k$ does not belong to the union of all the regions, we call the quantizer saturate. Thus a general quantizer may be given as

$$
q(k):= \begin{cases}k_{n} & k_{n} \leqslant k \\ k_{n-1} & k_{n-1} \leqslant k<k_{n} \\ \vdots & \\ 0 & 0 \leqslant k<k_{1} \\ -q(-k) & k<0,\end{cases}
$$

where $k_{n}=M$ is the upper boundary of the quantizer, which means the measure range of the quantizer is $[-M, M]$. The quantizer is saturate when $k \notin[-M, M] . \Delta:=k_{i}-k_{i-1}>0$ is called a quantization interval. A quantizer is called uniform if all its quantization intervals $\Delta$ are equal.

Note that there exists a dead region in some neighbourhood of the origin, where the quantization value is zero. This brings something to a quantization system, for instance, the hysteresis in real-time control system. But this is not what we want.

Here, we introduce the approach that is used in [17]:

$$
q_{\mu}(k):=\mu q\left(\frac{k}{\mu}\right),
$$

where $\mu>0$ is the so-called quantization factor. $q_{\mu}(k)$ is uniform if $q(k)$ is uniform.

Thus the range of the quantizer is $M \mu$ and the quantization interval is $\Delta \mu$. For an existing quantizer, we can choose a proper $\mu$ to deal with the saturate and the dead region of the quantizer.

The quantizers (2) considered in this paper are all uniform and are identical all the time. Of course, all the quantizers have the same quantization factor which is denoted by $\mu$ for the following use.

\section{Problem Statement}

Consider a system with $N$ agents, whose motion is modelled by a single integrator model:

$$
\dot{x}_{i}(t)=u_{i}(t), \quad i \in \ell=\{1,2, \ldots, N\},
$$

where $x_{i} \in R$ denotes the state of agent $i$ and $u_{i}$ is the input of agent $i$.

The communication topology of the considered multiagent system is presented by an undirected graph $G=(\ell, \varepsilon, A)$. Use $N_{i}$ denotes the communication set of agent $i$, which means if $(i, j) \in \varepsilon$, then $j \in N_{i}$.

Introducing the agreement control laws in [21-23],

$$
u_{i}=-\sum_{j \in N_{i}}\left(x_{i}-x_{j}\right) .
$$

For the nominal system without quantization, the closedloop state space description of the considered system is

$$
\dot{x}=u=-L x,
$$

where $L$ is the Laplacian matrix of $G$ and $x$ := $\left[x_{1}, x_{2}, \ldots, x_{N}\right]^{\mathrm{T}}$ is the vector of the states of all agents.

To state quantization, the closed-loop equation of the nominal system is $\dot{x}_{i}=-\sum_{j \in N_{i}}\left(q_{\mu}\left(x_{i}\right)-q_{\mu}\left(x_{j}\right)\right)$; then $\dot{x}=u=-L q_{\mu}(x)=-L \mu q(x / \mu)$, where $q_{\mu}(x)=$ $\left[q_{\mu}\left(x_{1}\right), q_{\mu}\left(x_{2}\right), \ldots, q_{\mu}\left(x_{N}\right)\right]^{\mathrm{T}}$ is the quantization of the state vector of agents. To input quantization, the closed-loop equation of the nominal system is $\dot{x}_{i}=-q_{\mu}\left(\sum_{j \in N_{i}}\left(x_{i}-x_{j}\right)\right)$; then $\dot{x}=q_{\mu}(u)=-q_{\mu}(L x)$.

One of the purposes of this paper is to give a practical strategy for updating $\mu$, so that the whole system can reach 
consensus. Take event-triggered strategy into consideration, which has been used in the multiagent systems without quantization in [22]. We use it in the multiagent systems with quantizers to generate state quantization and input quantization. For each case, update $\mu$ when an event $f=0$ holds to cope with the saturate and the subdivision of dead region of quantizers. According to the given event and the motion of agents, a sequence of time $t_{0}, t_{1}, \ldots$ is obtained and updating $\mu$ when $t=t_{s}$. Thus the sequence of time corresponding to a sequence of parameter $\mu\left(t_{0}\right), \mu\left(t_{1}\right), \ldots$ Between control updates and the quantization factor $\mu$ remains constant and equals to the last control update; rewrite the control law as

$$
\mu(t)=\mu\left(t_{s}\right), \quad t \in\left(t_{s}, t_{s+1}\right) .
$$

Note that $\mu$ is piecewise constant between the event times $t_{0}, t_{1}, \ldots$. Consider the following.

(1) States quantization: the control law is

$$
u(t)=-L q_{\mu\left(t_{s}\right)}(x), \quad t \in\left(t_{s}, t_{s+1}\right) .
$$

(2) Input quantization: the control law is

$$
u(t)=-q_{\mu\left(t_{s}\right)}(L x), \quad t \in\left(t_{s}, t_{s+1}\right) .
$$

Meanwhile, the block diagrams studied by this paper are depicted in Figure 1. Figures 1(a) and 1(b) show the block diagram of state quantization case and input quantization case, respectively. The main aim of the following sections is to give the update strategy of the quantization factors and steps for achieving consensus.

Define two designations so that we can use them in the following sections:

(1) $z=\left[z_{1}, z_{2}, \ldots, z_{N}\right]^{\mathrm{T}}:=L x$; obviously, $z_{i}$ just relates to the neighbourhoods of agent $i$;

(2) $\alpha:=\mu \Delta$, which means the quantization interval of quantizer (2).

Obviously, $\alpha>0$ because $\mu>0$ and $\Delta>0$.

\section{Main Results}

In this section, consensus strategies for multiagent systems with state quantization and input quantization are demonstrated, respectively.

\subsection{State Quantization. Rewrite the control law as}

$$
u(t)=-L q_{\mu_{s}}(x(t))=-L \mu_{s} q\left(\frac{x(t)}{\mu_{s}}\right)
$$

where $\mu_{s}$ remains constant during $\left[t_{s}, t_{s+1}\right)$. Thus the closedloop system is presented by

$$
\dot{x}(t)=-L q_{\mu_{s}}(x(t))=-L \mu_{s} q\left(\frac{x(t)}{\mu_{s}}\right) .
$$

And it is easy to show that the average of the states remains constant all the time.
For system (10), a candidate ISS Lyapunov function [24] is

$$
V=\frac{1}{2} x^{\mathrm{T}} L x
$$

Note that $z:=L x$. Then the time derivation of $V$ is

$$
\begin{aligned}
\dot{V} & =x^{\mathrm{T}} L \dot{x} \\
& =-x^{\mathrm{T}} L L \mu\left[q\left(\frac{x}{\mu}\right)+\frac{x}{\mu}-\frac{x}{\mu}\right] \\
& \leqslant-z^{\mathrm{T}} z+x^{\mathrm{T}} L L \alpha \\
& \leqslant-\|z\|^{2}+\|z\|\|L\| \alpha \\
& =-\|z\|(\|z\|-\|L\| \alpha) .
\end{aligned}
$$

With arbitrary positive integer $\sigma<p-1$ (where $p$ satisfies $p \Delta=M)$, when

$$
\|z\| \geqslant(1+\sigma)\|L\| \alpha
$$

holds, we have

$$
\dot{V} \leqslant-\sigma\|z\|\|L\| \alpha
$$

which is negative for $\|z\| \neq 0$. Thus we can change $\mu$ by the event

$$
f(x)=\|z\|-(1+\sigma)\|L\| \alpha=0 .
$$

This choice is motivated by the analysis of the subdivision of the dead region above. Thus the quantization distance would tend towards origin.

Lemma 1. Consider a multiagent system with the state equation (10), and assume $G$ is connected. Define two ellipsoids:

$$
\begin{aligned}
& \Omega_{1}(\mu)=\left\{x: x^{T} L x \leqslant\|L\| M^{2} \mu^{2}\right\}, \\
& \Omega_{2}(\mu)=\left\{x: x^{T} L x \leqslant\|L\|(1+\sigma)^{2} \alpha^{2}\right\} .
\end{aligned}
$$

For arbitrary positive $\sigma<p-1$, each state starts inside $\Omega_{1}$ but outside $\Omega_{2}$ will enter into $\Omega_{2}$ in finite time which is upper bounded by

$$
\tau=\frac{p^{2}-(1+\sigma)^{2}}{\|L\| \sigma(1+\sigma)}
$$

where $p \Delta=M$. That is, for any initial position in annular section made up by $\Omega_{1}$ and $\Omega_{2}$, event (15) will occur in a finite time no more than $\tau$.

Proof. It is obvious that $\Omega_{2} \subset \Omega_{1}$.

Consider $E_{1}=\|L\| M^{2} \mu^{2}$ and $E_{2}=\|L\|(1+\sigma)^{2} \alpha^{2}$ as the maximum energy of the states locate in $\Omega_{1}$ and $\Omega_{2}$, respectively. And $E_{1}>E_{2}$ because $\sigma<p-1$.

Instead of $\|z\|$ in (14) with (13), we have

$$
\dot{V} \leqslant-\|L\|^{2} \sigma(1+\sigma) \alpha^{2} .
$$




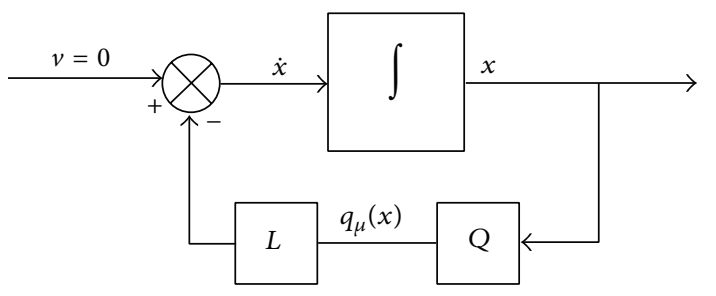

(a) State quantization case

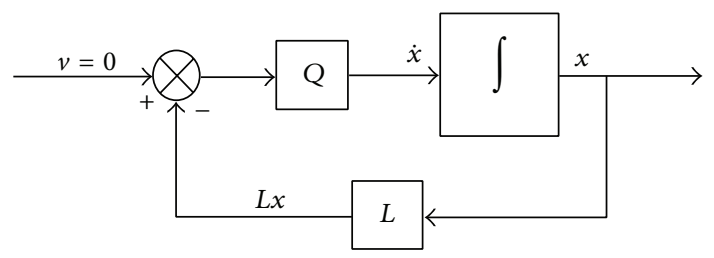

(b) Input quantization case

FIGURE 1: Block diagrams of the problems this paper studies.

The absolute value of (18) is

$$
|\dot{V}| \geqslant\|L\|^{2} \sigma(1+\sigma) \alpha^{2} .
$$

For closed-loop system (10), according to inequation (19), when (13) holds and the initial state is located inside $\Omega_{1}$ but outside $\Omega_{2}$, the defined energy decreases in a speed that is lower bounded by $\|L\|^{2} \sigma(1+\sigma) \alpha^{2}$. Event (15) occurs when the states reach the bound of $\Omega_{2}$; meanwhile (13) does not hold. Well, the worst condition is that the initial state is on the boundary of ellipsoid $\Omega_{1}$ and the energy of the system decreases in the speed of $\|L\|^{2} \sigma(1+\sigma) \alpha^{2}$ all the time during a constant $\mu$. In this condition, the state will enter into ellipsoid $\Omega_{2}$ in a finite time:

$$
\tau=\frac{M^{2} \mu^{2}-(1+\sigma)^{2} \alpha^{2}}{\|L\| \sigma(1+\sigma) \alpha^{2}} .
$$

Since $p \Delta=M, \tau$ can be simplified as

$$
\tau=\frac{p^{2}-(1+\sigma)^{2}}{\|L\| \sigma(1+\sigma)} .
$$

That is, if $x\left(t_{0}\right) \in \Omega_{1}$, then $x\left(t_{0}+\tau\right) \in \Omega_{2}$.

This completes the proof.

Theorem 2. Assume that $\rho:=\sqrt{M^{2} /(1+\sigma)^{2} \Delta^{2}}$ and $\bar{\rho}:=$ $1 / \rho$. Closed-loop system (10) can reach consensus under the following steps.

(i) Search the upper bound: set $u=0$; then $\dot{x}=0$; this indicates the system is open-loop, and in this process, the states remain constant. Set $\mu\left(t^{0}\right)=1$ for $t \in[0, T)$, where $T$ depends on the operating speed of the processor and the quantizer, let $\mu\left(t^{1}\right)=\rho \mu\left(t^{0}\right)=\rho, \mu\left(t^{2}\right)=\rho^{2}$, and so on. There exists a time $t^{m}$ that $x^{T} L x<\|L\| M^{2} \mu^{2}\left(t^{m}\right)$ holds. Thus we know the upper bound.

(ii) Search the lower bound: once condition ( $i)$ is satisfied, if $m \neq 0, \mu\left(t^{m}\right)=\rho^{m}$, and $x^{T} L x \geqslant\|L\|(1+\sigma)^{2} \mu^{2}\left(t^{m}\right) \Delta^{2}$ are obviously satisfied, then skip this step. If $m=0$, then let $\mu\left(t^{1}\right)=$ $\bar{\rho} \mu\left(t^{0}\right)=\bar{\rho}, \mu\left(t^{2}\right)=\bar{\rho}^{2}$, and so on. Then there is a time $t^{n}$ that $x^{T} L x \geqslant\|L\|(1+\sigma)^{2} \alpha^{2}$ holds. Thus we know the lower bound.

From the above two steps, we can pick a $\mu_{0}$ that guarantees the initial states located inside the ellipsoid $\Omega_{1}$ but outside the ellipsoid $\Omega_{2}$.

(iii) Set $u=-L q_{\mu_{i}}(x)$, then update $\mu$ by $\mu_{i}=\bar{\rho}^{i} \mu_{0}$ when event (15) is satisfied repeatedly.
Since $\sigma<p-1$, so $\bar{\rho}<1$. Thus $x^{\mathrm{T}} L x \rightarrow 0$ as $i \rightarrow \infty$. From Lemma 1, we know $i \rightarrow \infty$ when $t \rightarrow \infty$, which means the considered closed-loop system is asymptotically convergent with $t \rightarrow \infty$, then consensus is achieved.

4.2. Input Quantization. For input quantization case, the control law of the closed-loop system is

$$
u(t)=-L x=-z .
$$

Then the closed-loop system is

$$
\dot{x}(t)=q_{\mu}(u(t))=-\mu q\left(\frac{z}{\mu}\right) .
$$

In this case, we also choose the Lyapunov function as

$$
V=\frac{1}{2} x^{\mathrm{T}} L x
$$

Then we have

$$
\begin{aligned}
\dot{V} & =x^{\mathrm{T}} L \dot{x} \\
& \leqslant-\|z\|^{2}+\|z\| \alpha \\
& =-\|z\|(\|z\|-\alpha) .
\end{aligned}
$$

This implies that if positive integer satisfies $\sigma<p-1$ (where $p$ has the same meaning as the state quantization condition), then

$$
\begin{gathered}
\|z\| \geqslant(1+\sigma) \alpha \\
\Longrightarrow \dot{V} \leqslant-\|z\| \sigma \alpha .
\end{gathered}
$$

Note that only quantized measurements of the inputs are available. The event should be

$$
f(q)=\left\|q_{\mu}(z)\right\|-(1+\sigma) \alpha=0 .
$$

Lemma 3. Consider a multiagent system with the state equation (23), and assume that its Laplacian $G$ is connected. Define two ellipsoids:

$$
\begin{aligned}
& \Omega_{1}(\mu)=\left\{x: x^{T} L x \leqslant \frac{M^{2} \mu^{2}}{\|L\|}\right\}, \\
& \Omega_{2}(\mu)=\left\{x: x^{T} L x \leqslant \frac{(1+\sigma)^{2} \alpha^{2}}{\|L\|}\right\} .
\end{aligned}
$$


For arbitrary positive integer $\sigma<p-1$, each state starting inside $\Omega_{1}$ but outside $\Omega_{2}$ will enter into $\Omega_{2}$ in finite time that is upper bounded by

$$
\tau=\frac{p^{2}-(1+\sigma)^{2}}{\|L\| \sigma(1+\sigma)},
$$

where $p \Delta=M$. That is, for any state in $\Omega_{1}$, event (28) will occur in a finite time no more than $\tau$.

Proof. It is obvious that $\Omega_{2} \subset \Omega_{1}$.

Consider $E_{1}=M^{2} \mu^{2} /\|L\|$ and $E_{2}=(1+\sigma)^{2} \alpha^{2} /\|L\|$ to be the maximum energy of the states located in $\Omega_{1}$ and $\Omega_{2}$, respectively. And $E_{1}>E_{2}$ because $\sigma<p-1$.

Instead of $\|z\|$ in (27) with (26), we have

$$
\dot{V} \leqslant-\sigma(1+\sigma) \alpha^{2}
$$

The absolute value of (31) is

$$
|\dot{V}| \geqslant \sigma(1+\sigma) \alpha^{2} .
$$

For closed system (23), from inequation (32), when (26) holds and the initial state locates inside $\Omega_{1}$ but outside $\Omega_{2}$, the defined energy decreases in a speed that is lower bounded by $\sigma(1+\sigma) \alpha^{2}$. The states enter $\Omega_{2}$ once when event (28) occurs; meanwhile inequality (26) does not hold. Well, the worst condition is that the initial state is at the boundary of ellipsoid $\Omega_{1}$, and the energy of the system decreases in the speed of $\sigma(1+\sigma) \alpha^{2}$ all the time for a constant $\mu$. In this condition, the state will enter into ellipsoid $\Omega_{2}$ in a finite time:

$$
\tau=\frac{M^{2} \mu^{2}-(1+\sigma)^{2} \alpha^{2}}{\|L\| \sigma(1+\sigma) \alpha^{2}} .
$$

Since $p \Delta=M, \tau$ can be simplified as

$$
\tau=\frac{p^{2}-(1+\sigma)^{2}}{\|L\| \sigma(1+\sigma)}
$$

that is, if $x\left(t_{0}\right) \in \Omega_{1}$, then $x\left(t_{0}+\tau\right) \in \Omega_{2}$.

This completes the proof.

Theorem 4. Assume that $\rho:=\sqrt{M^{2} /(1+\sigma)^{2} \Delta^{2}}$ and $\bar{\rho}:=$ $1 / \rho$. Closed-loop system (23) can reach consensus under the following steps.

(i) Search the upper bound: set $u=0$; then $\dot{x}=0$; this indicates that the system is open-loop, and in this process, the states remain constant. Set $\mu\left(t^{0}\right)=1$ for $t \in[0, T)$, where $T$ depends on the operating speed of the processor and the quantizer; let $\mu\left(t^{1}\right)=\rho \mu\left(t^{0}\right)=\rho, \mu\left(t^{2}\right)=\rho^{2}$, and so on. Then there exists a time $t^{m}$ that $x^{T} L x<M^{2} \mu^{2}\left(t^{m}\right) /\|L\|$ holds. Thus we know the upper bound.

(ii) Search the lower bound: once condition ( $i)$ is satisfied, if $m \neq 0, \mu\left(t^{m}\right)=\rho^{m}$, and $x^{T} L x \geqslant(1+\sigma)^{2} \mu^{2} \Delta^{2} /\|L\|$ are obviously satisfied, then skip this step. If $m=0$, then let $\mu\left(t^{1}\right)=$ $\bar{\rho} \mu\left(t^{0}\right)=\bar{\rho}, \mu\left(t^{2}\right)=\bar{\rho}^{2}$, and so on. Then there is a time $t_{n}$ that $x^{T} L x \geqslant(1+\sigma)^{2} \alpha^{2} /\|L\|$ holds. Thus we know the lower bound.
From the above two steps, we can pick a $\mu_{0}$ that guarantees the initial states locating inside the ellipsoid $\Omega_{1}$ but outside the ellipsoid $\Omega_{2}$.

(iii) Set $u=-q_{\mu}(L x)$; then update $\mu$ by $\mu=\bar{\rho}^{i} \mu_{0}$ when event (28) occurs.

Since $\sigma<p-1$, so $\bar{\rho}<1$. Thus $x^{\mathrm{T}} L x \rightarrow 0$ as $i \rightarrow \infty$. From Lemma 3, we know $i \rightarrow \infty$ when $t \rightarrow \infty$, which means the considered closed-loop system is asymptotically convergent with $t \rightarrow \infty$; then consensus is achieved.

4.3. Discussion. From the deductions of event (15) and event (28), it is easy to get that the Laplacian matrix $L$ satisfies $L=L^{\mathrm{T}}$, which means the Laplacian matrix of the considered system should be symmetrical. In other words, the consensus strategy can work well when the Laplacian matrixes of the systems with weighted topologies are symmetrical.

\section{Examples}

The results of this paper are illustrated by the following example, in which we take state quantization case into consideration.

Consider a first-order system with four agents whose Laplacian matrix is given by

$$
L=\left[\begin{array}{cccc}
1 & -1 & 0 & 0 \\
-1 & 3 & -1 & -1 \\
0 & -1 & 2 & -1 \\
0 & -1 & -1 & 2
\end{array}\right]
$$

Think of the state quantization case. Four agents start from a random position with control law (10) and following Theorem 2. Assume that $x_{0}=\left[\begin{array}{llll}0.5 & 0 & 1 & 0.2\end{array}\right]$. Set $M=5$ and $\mu\left(t_{0}\right)=1$.

Figure 2 shows the case of the closed-loop system with 10-bit quantizers, and Figures 2(a) and 2(b) the case where $\sigma=20$ and $\sigma=200$, respectively. Figure 3 shows the case of the closed-loop system with 16-bit quantizers, and Figures 3(a) and 3(b) show the case where $\sigma=20$ and $\sigma=$ 200 , respectively. Figure 4 shows the results of four agents that have the same topology but under consensus strategy (4).

Note that in the two cases discussed above, the quantization interval $\Delta$ is $1 / 1023$ and $1 / 65535$, respectively. Because of the quantization interval, it is possible to produce an error for the initial states quantized value, and the bigger the quantization interval is, the bigger the error is. Just like the existence of the initial quantization error, there may be a quantization error in the end. In cases without quantizers, [22] shows if the graph of a multiagent system is connected, following the control law (5), the states would converge to their initial average. So with quantizers in the system, states would converge to some constant values close to their initial average with an error, and the bigger the quantization interval is, the bigger the error is.

Figures 2(a) and 2(b) depict how the parameter $\sigma$ effects the accuracy of the final states of agents. And it shows that the final states have high accuracy for a bigger $\sigma$, but more time is cost. Comparing Figures 2(a) with 3(a) and 2(b) with 3(b), it is 


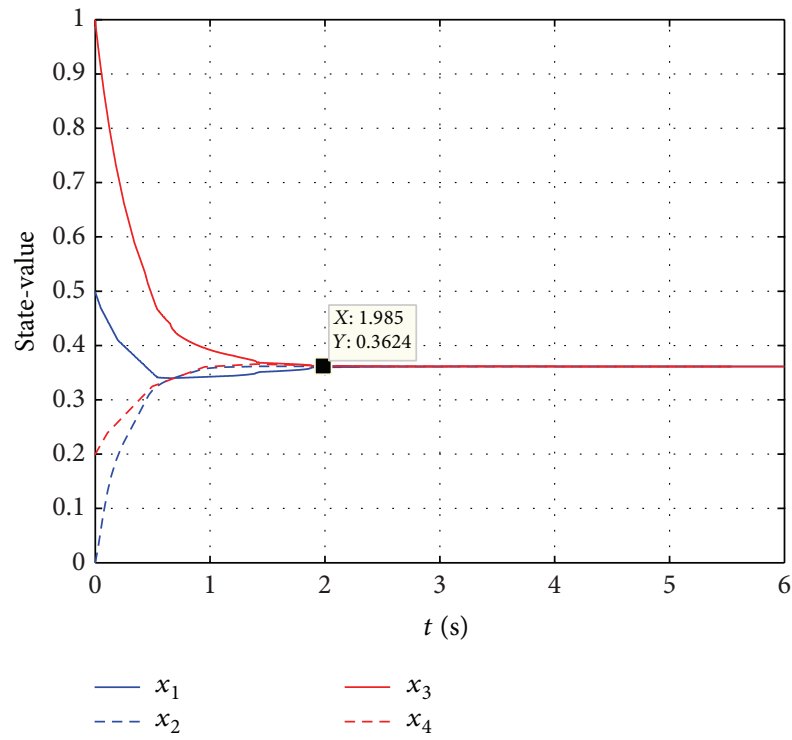

(a) For $\sigma=20$

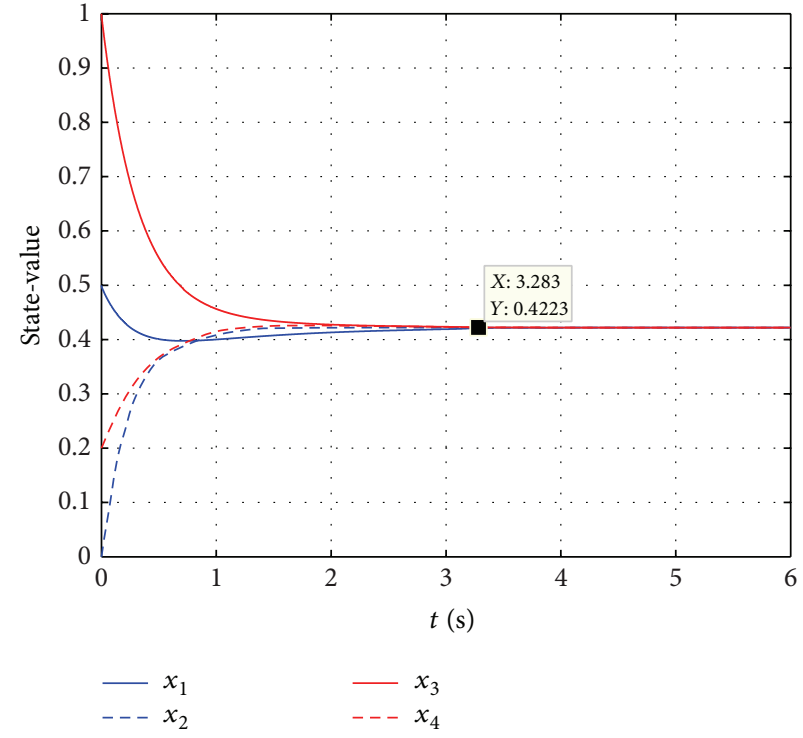

(b) For $\sigma=200$

FIGURE 2: Four agents evolve under (10), (15) in the state quantization case. With $\Delta=1 / 1023$, converge closely to the initial average of the initial quantization of each agents.

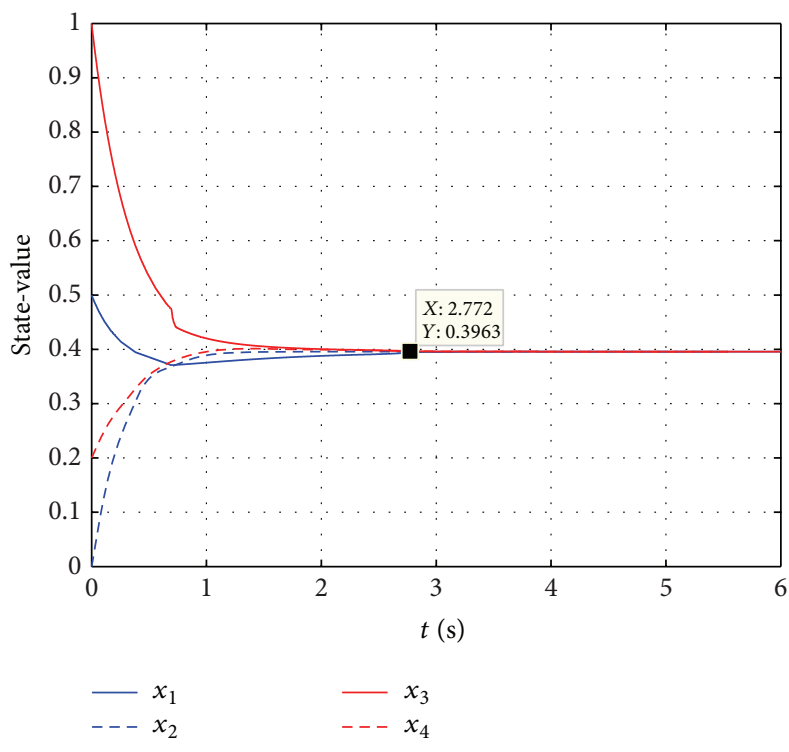

(a) For $\sigma=20$

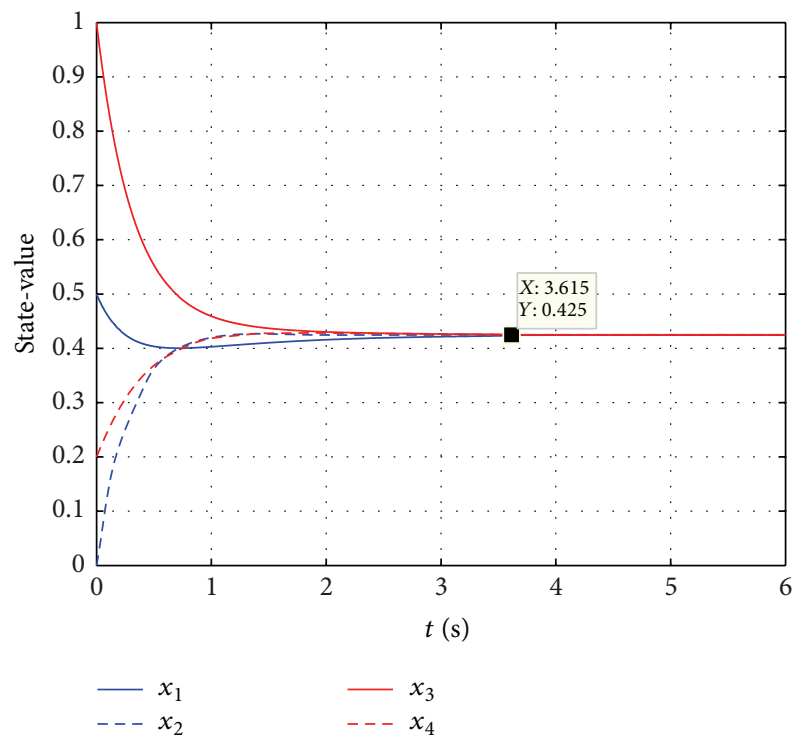

(b) For $\sigma=200$

FIGURE 3: Four agents evolve under (10), (15) in the state quantization case. With $\Delta=1 / 65535$, converge closely to the initial average of the initial quantization of each agents.

easy to know that accuracy of the final states would be higher with smaller quantization interval $\Delta$ when $\sigma$ keeps constant, which meets the above analysis perfectly. This also indicates that the effect of $\Delta$ is not so much obvious for a bigger $\sigma$. So in practice, we could use bigger $\sigma$ and a not too small quantization interval $\Delta$ (for instance, the less-bits ADT) to reach satisfied final accuracy and thus could save some time and reduce the expense.
Comparing Figure 4 with Figure 2 and Figure 3, it is obvious that the former always takes more time than the latter when the systems begin with the same initial condition and reach consensus in the end. What is more worth mentioning is the quantized values transferred or used when employing the strategies that this paper proposed, which means the communication and compute burden would be lower under the strategies that this paper proposed. 


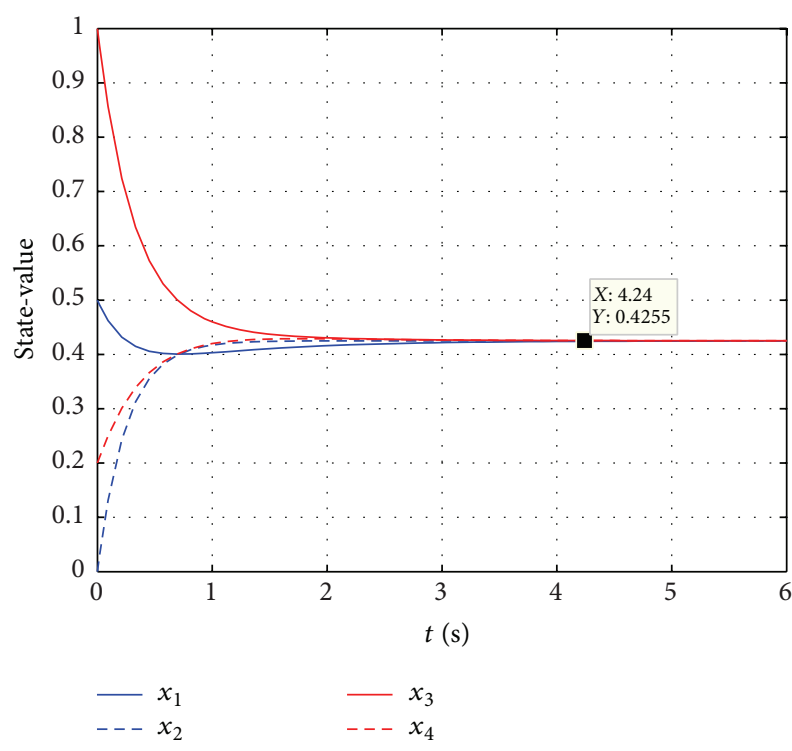

FIGURE 4: Four agents of system (3) evolve under consensus strategy (4).

\section{Conclusion}

This paper investigates the quantized consensus problem of a first-order multiagent system. Both the state quantization and the input quantization are considered. The convergence steps for both cases are given. A simulation result supports the results of this paper perfectly. Future work may be focused on the application of some other effective quantizers in multiagent systems and the solution of the quantized consensus problems of multiagent systems by distributed strategies. Furthermore, systems with directed topology are more practical. So the application of the proposed strategy in these systems is more interesting but more challenging.

\section{Conflict of Interests}

The authors declare that there is no conflict of interests regarding the publication of this paper.

\section{References}

[1] A. Hyvärinen, "Fast and robust fixed-point algorithms for independent component analysis," IEEE Transactions on Neural Networks, vol. 10, no. 3, pp. 626-634, 1999.

[2] M. Chen, S. S. Ge, and B. V. E. How, "Robust adaptive neural network control for a class of uncertain MIMO nonlinear systems with input nonlinearities," IEEE Transactions on Neural Networks, vol. 21, no. 5, pp. 796-812, 2010.

[3] K. Li, J. Cao, and F. Yu, "Study on the nonsingular problem of fractional-order terminal sliding mode control," Mathematical Problems in Engineering, vol. 2013, Article ID 523251, 7 pages, 2013.

[4] T. Sangpet and S. Kuntanapreeda, "Force control of an electrohydraulic actuator using a fractional-order controller," Asian Journal of Control, vol. 15, no. 3, pp. 764-772, 2013.
[5] K. G. Sheela and S. N. Deepa, "Review on methods to fix number of hidden neurons in neural networks," Mathematical Problems in Engineering, vol. 2013, Article ID 425740, 11 pages, 2013.

[6] S. Lv, J. Tian, and S. Zhong, "Delay-dependent stability analysis for recurrent neural networks with time-varying delays," Mathematical Problems in Engineering, vol. 2012, Article ID 910140, 14 pages, 2012.

[7] Y. Liu and Z. Geng, "Finite-time optimal formation control for second-order multiagent systems," Asian Journal of Control, vol. 16, no. 1, pp. 138-148, 2014.

[8] W. Ren, R. W. Beard, and E. M. Atkins, "Information consensus in multivehicle cooperative control," IEEE Control Systems Magazine, vol. 27, no. 2, pp. 71-82, 2007.

[9] X. Wang and Y. Hong, "Finite-time consensus for multi-agent networks with second-order agent dynamics," in Proceedings of the 17th IFAC World Congress International Federation of Automatic Control, pp. 15185-15190, 2008.

[10] Z.-W. Liu, Z.-H. Guan, and H. Zhou, "Impulsive consensus for leader-following multiagent systems with fixed and switching topology," Mathematical Problems in Engineering, vol. 2013, Article ID 762861, 10 pages, 2013.

[11] K. You, L. Xie, S. Sun, and W. Xiao, "Quantized filtering of linear stochastic systems," Transactions of the Institute of Measurement and Control, vol. 33, no. 6, pp. 683-698, 2011.

[12] K. You, W. Su, M. Fu, and L. Xie, "Attainability of the minimum data rate for stabilization of linear systems via logarithmic quantization," Automatica, vol. 47, no. 1, pp. 170-176, 2011.

[13] M. Fu and L. Xie, "The sector bound approach to quantized feedback control," IEEE Transactions on Automatic Control, vol. 50, no. 11, pp. 1698-1711, 2005.

[14] D. F. Delchamps, "Stabilizing a linear system with quantized state feedback," IEEE Transactions on Automatic Control, vol. 35, no. 8, pp. 916-924, 1990.

[15] X. Feng and K. A. Loparo, "Active probing for information in control systems with quantized state measurements: a minimum entropy approach," IEEE Transactions on Automatic Control, vol. 42, no. 2, pp. 216-238, 1997.

[16] J. Sur and B. E. Paden, "State observer for linear time-invariant systems with quantized output," Journal of Dynamic Systems, Measurement and Control, Transactions of the ASME, vol. 120, no. 3, pp. 423-426, 1998.

[17] D. Liberzon, "Hybrid feedback stabilization of systems with quantized signals," Automatica, vol. 39, no. 9, pp. 1543-1554, 2003.

[18] G. Chen, F. L. Lewis, and L. Xie, "Finite-time distributed consensus via binary control protocols," Automatica, vol. 47, no. 9, pp. 1962-1968, 2011.

[19] T. Li, M. Fu, L. Xie, and J.-F. Zhang, "Distributed consensus with limited communication data rate," IEEE Transactions on Automatic Control, vol. 56, no. 2, pp. 279-292, 2011.

[20] F. Ceragioli, C. de Persis, and P. Frasca, "Discontinuities and hysteresis in quantized average consensus," Automatica, vol. 47, no. 9, pp. 1916-1928, 2011.

[21] J. A. Fax and R. M. Murray, "Graph laplacians and stabilization of vehicle formation," in Proceedings of the 15th International Federation of Automatic Control World Congress, 2002.

[22] D. V. Dimarogonas and K. H. Johansson, "Event-triggered control for multi-agent systems," in Proceedings of the 48th IEEE Conference on Decision and Control held jointly with 28th Chinese Control Conference (CDC/CCC '09), pp. 7131-7136, December 2009. 
[23] R. Olfati-Saber and R. M. Murray, "Consensus problems in networks of agents with switching topology and time-delays," IEEE Transactions on Automatic Control, vol. 49, no. 9, pp. 15201533, 2004.

[24] E. D. Sontag and Y. Wang, "On characterizations of the inputto-state stability property," Systems \& Control Letters, vol. 24, no. 5, pp. 351-359, 1995. 


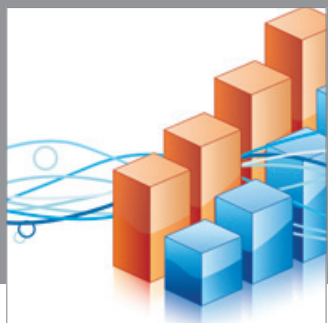

Advances in

Operations Research

mansans

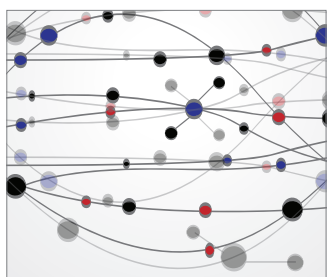

The Scientific World Journal
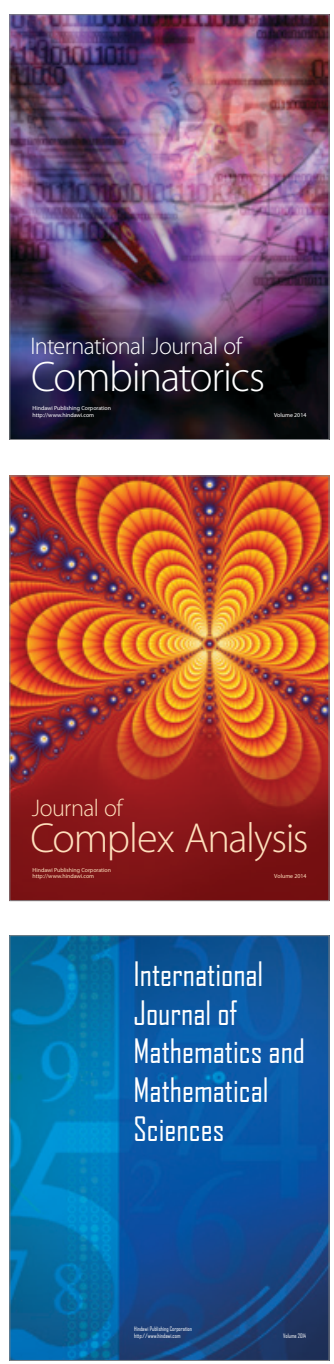
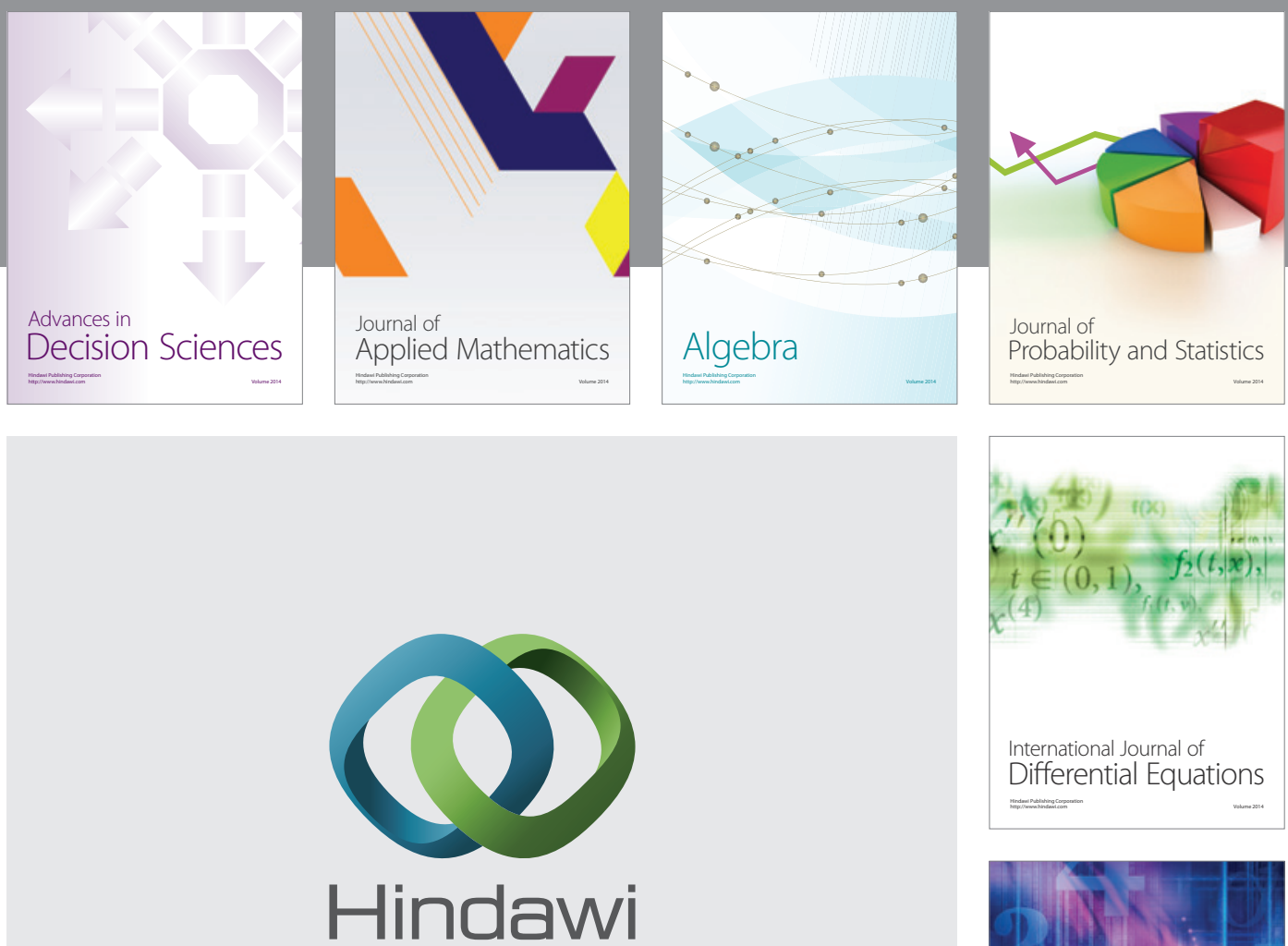

Submit your manuscripts at http://www.hindawi.com
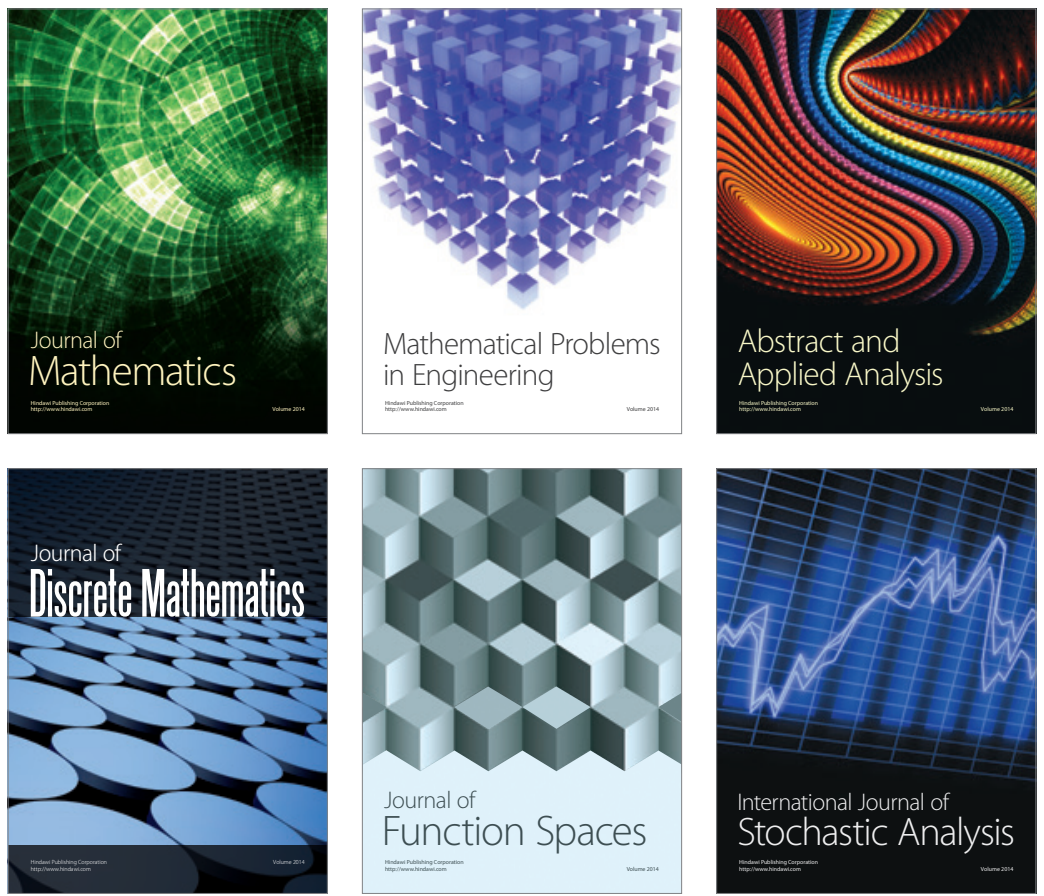

Journal of

Function Spaces

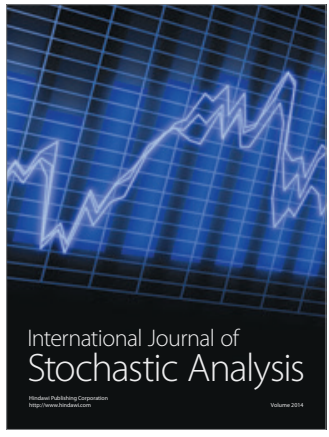

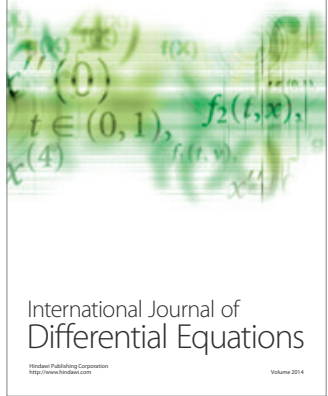
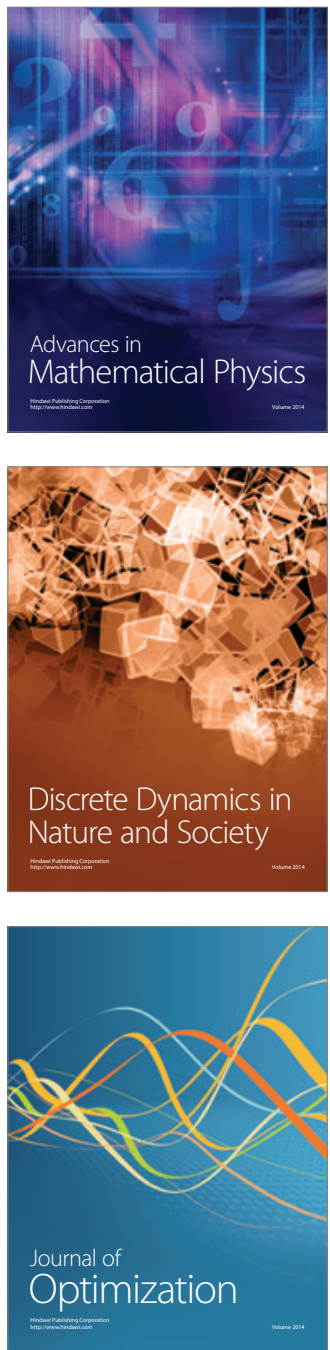